\title{
Development of the Sustainable Technology Balance Sheet (STBS) - A generic method to assess the sustainability of renewable energy technologies
}

\author{
Alan C Brent ${ }^{1, *}$, Wildri D Peach ${ }^{2,3}$, William Stafford ${ }^{2}$ \\ ${ }^{1}$ Centre for Renewable and Sustainable Energy Studies, School of Public Leadership, Stellenbosch \\ University, Stellenbosch, South Africa \\ ${ }^{2}$ Sustainable Energy Futures, Natural Resources and the Environment, Council for Scientific and Industrial \\ Research, Pretoria, South Africa \\ ${ }^{3}$ Graduate School of Technology Management, University of Pretoria, Pretoria, South Africa \\ *Corresponding author. Tel: +27 21881 3952, Fax: +27 21881 3294, E-mail: acb@sun.ac.za
}

\begin{abstract}
The impacts of technologies on sustainability have to be assessed through structured approaches to provide decision-makers with strategic information. Traditional technology assessment methods can be complex and highly resource intensive with long lead times; consequently, the applications of these methods are limited, especially in Africa. Where these methods have been applied, the conclusions that are generated are also not always effectively communicated, which leads to limited buy-in from stakeholders. The paper therefore proposes a generic rapid technology assessment framework and implementation process that utilises a popular method that has been modified to include sustainability factors and a systems approach, while remaining simple and intuitive: the Sustainable Technology Balance Sheet (STBS). The method addresses technology assessment from a qualitative view by including sustainability criteria developed through stakeholder engagement and technical factors through expert opinion, while inducing a life cycle approach to ensure system awareness. A case study approach, using a bioenergy value chain, is used to demonstrate the developed STBS method.
\end{abstract}

Keywords: Sustainability assessment, technology assessment, Africa.

\section{Introduction}

Energy is closely linked to the sustainable development paradigm. The impact of energy technologies can include climate change, which is associated with excess use of energy, and poverty, due to a lack of access to energy. Solutions to these sustainability problems may be achieved by using new technologies, such as renewable energy technologies (RETs), that reduce pollution and, in some instances, provide development opportunities. Such solutions can, however, only be achieved if the correct technology strategies are followed by effectively assessing and communicating viable options to policy makers.

A key issue for sustainable development is the various implications of the extraction, generation and use of energy that must be evaluated in a comprehensive manner. As the worldwide demand for energy resources increases so too does the diverse range of impacts that occur over the respective energy value chains relating to the various acquisition and operational activities as well as from the utilised technologies.

In attempting to address the sustainable development challenges that technology presents, structured approaches and firm methodologies must be developed and implemented as a prerequisite to ensure the comprehension and coordination to reach intended outcomes. Technology assessment methods can provide the basis for this development [1].

\section{Development of the Sustainable Technology Balance Sheet (STBS) method}

The conventional Technology Balance Sheet (TBS) is one technology assessment method that has been utilised effectively [2]. It is a graphical representation of the interrelationships, interdependence and reliance between the factors of technologies, processes, products, and markets. The foundation for the TBS is the relative relationship between these four factors. 
Originally the relationship was based on economics and how the factors met each other's demands [3].

The simplistic logic of the framework, which is indicative of the relationship between the factors considered, makes use of a simple matrix to relate two specific factors. This is then augmented by other matrices to enhance the relationship or connection between factors while still retaining the straightforward logic behind each matrix (see Fig. 1).

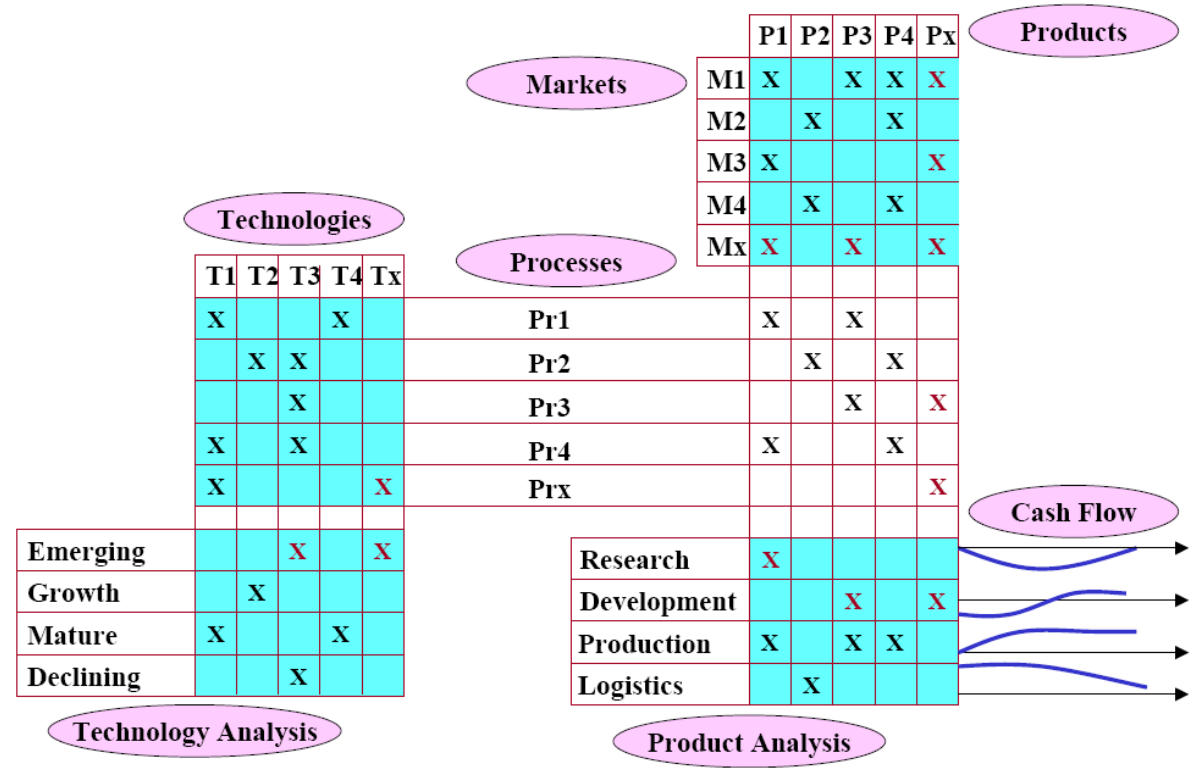

Fig. 1. The conventional Technology Balance Sheet method [3].

Within this framework, when a new technology is incorporated into an existing or new process to produce a product, which meets an established market demand, or creating a whole new market niche. The technology thus acts as a driver for new products and processes due to its enabling characteristics needed for existing products and process. This defines the connection between the four factors through the interconnected nature of the factors [4].

The TBS is a business-orientated tool designed to aid managers in the technology decision making process. The tool intends to facilitate and guide an enterprise through a technology assessment process towards a clearer understanding of the conclusions ultimately produced by the framework. The enlightenment generated by the process is often more valuable than the outcome obtained. This would include a better understanding of how organisational structures relate to each other and how operational flows affect the business, both by means of a greater internal and external awareness. Nevertheless, the TBS will still be a communication tool, to effectively communicate the outcomes to those not involved in the process, as well as nontechnical stakeholders who will be able to draw logical conclusions and intrinsically generate the correct answer, which is so important for personal buy in and ultimate project success [4].

The TBS answers the questions of "where we are" as a business looking at technology and provides strategic direction by answering "where to go" as well as "where to get out" by making use of technology s-curves and analysing where a technology is located in the technology life cycle [3]. The TBS indicates the forces at work within the techno-economic system. These forces manifest themselves within the organisation as opposing directional force, simply as a push or a pull [3]; they are produced by different elements within the factors. A market force can be described as a pulling force pulling business output towards the 
market demand, be it though desired products, which occur only once the force has been transmitted to the processes to generate the capabilities within the business. However to produce the products and develop these processes only occurs once the pull force has been transferred to the technology factor to grow, develop and provide the methodologies required to generate the processes required to create the products to meet the market need. If one considers technology as a push force we can experience a force from a new technological invention or development pushing along new capabilities and new processes, which can lead to new or advanced products and through their existence create new markets or change the dynamics of existing ones. These two forces can have a feedback effect on the entire system as the process and capabilities continue to grow and so a type of causal loop system has been created.

The TBS provides organisational value by highlighting the drivers at work within the organisation and how these can be manipulated to be successful in meeting the business goals. As one becomes more aware of how each factor relates to the others, one is more able to grasp their impact. This would not only be unique to being economically successful, as is the traditional intent of the TBS, but by reviewing the intent, aligning the point of view and reassessing the goals we will be able to use the simple TBS framework to meet any desired outcomes; for the problem at hand, to address sustainability while critically assessing different energy technologies. Therefore, sustainability can be introduced in the TBS by making use of the principles or criteria used for the assessment of environmental, social and economic sustainability and would include those applied in the broad sustainability body of knowledge [5] and refined through a needs analysis that comprises stakeholder engagement.

\subsection{STBS framework and Implementation Process}

A sustainable technology assessment tool has subsequently been developed [6] and consists of two parts: first, the Sustainable Technology Balance Sheet (STBS) which is a rapid technology assessment and communication framework; and second, an integral part that is referred to as the Implementation Process, which is a structured method through which the relevant stakeholders can be engaged and qualitative data can be obtained. Each part consists of specific methodologies and underlying logic, which can be summarised as shown in Fig. 2 .

The Implementation Process consists of four steps initiated by a facilitator during stakeholder engagement workshops to generate the information needed to populate the STBS, create system awareness and project enlightenment among these stakeholders [6]:

- Step 1a: Value Chain Generation: through a life cycle analysis and by the investigation of the product/process life cycle to generate, firstly a generic value chain and secondly, once the components of the value chain are validated, a case specific process value chain is generated.

- Step 1b: Sustainability Criteria Development: Sustainability aspects addressed by stakeholder engagement and literature review, which is done concurrently during the initial engagement stages. Once systems-thinking has been instilled, discussions surrounding the creation of specific Sustainability Criteria may be fulfilled. This would reaffirm the stakeholders' intentions toward sustainability.

- Step 2: Technology and Process Awareness: Achieved through the creation of inputprocess-output diagrams, which indicate process linkages known as Technology Super Structures. This is done for each one of the value chain components indicated by the dashed rings of Fig. 2. A short discussion surrounding the grouping or indexing of Sustainability Criteria into sectors may also be accomplished. 
- Step 3: STBS Development: The utilisation of the generated information and understanding to populate the STBS so as to formalise the information and to communicate conclusions accurately.

- Step 4: Strategic Direction and Conclusion Analysis the presentation of STBS outcomes to relevant stakeholders is of vital importance. This new impetus, created by the indicated strategic direction, needs to be subscribed to and further investigations can be made in an enlightened and qualified direction. These investigations can include, amongst others, multi-criteria decision analysis (MCDA) trees and life cycle analysis (LCA) studies to add more rigour to the indicated outcomes and strategic conclusions.

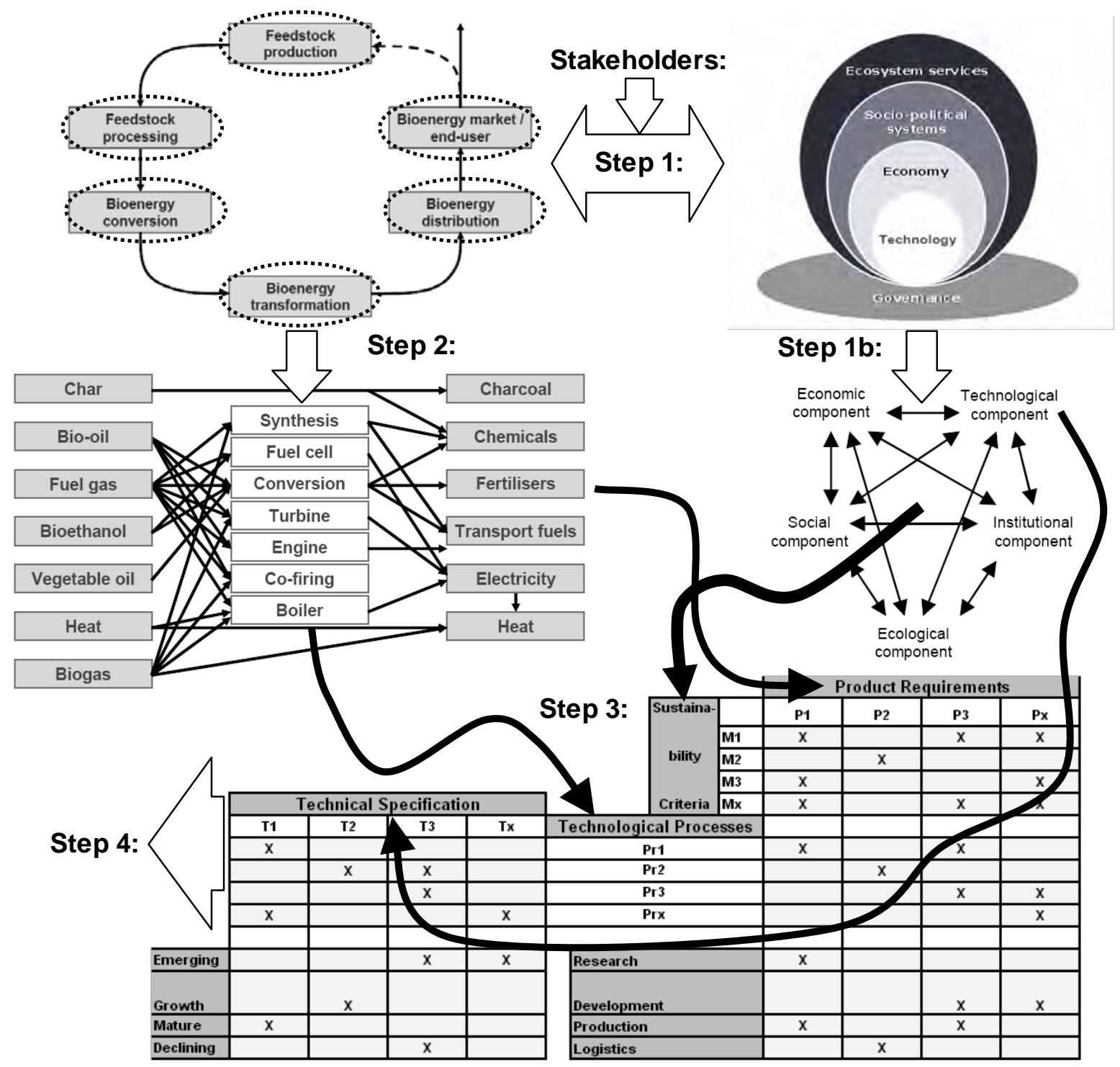

Fig. 2. The Implementation Process of the Sustainable Technology Balance Sheet (STBS).

\section{A case study to investigate the STBS method}

A case study approach was used to understand and test the developed STBS method. The case is of the Working for Energy (WfE) programme, which is an initiative of the South African Department of Water Affairs and Forestry (DWAF) to utilise waste invasive alien biomass as an energy source effectively adding value such as job creation and energy resources to the Working for Water projects through new Public Private Partnerships (PPPs). The need to 
assess suitable technologies for these PPPs was identified and the STBS tool was deemed as a suitable option.

The STBS was firstly discussed informally among a group of energy technology analysis experts to generate relevant information and determine the key stakeholders, which can be utilised during the development of the tool. These meetings then became provided feedback of developments and obstacles to the various experts and stakeholders.

Finally, a formal presentation of the proposed STBS tool was made during a workshop to experts, which included members of the national DWAF and other stakeholders at which time further inputs could be given. The consequence was a process of conceptualisation of a modified TBS, making use of a reiterative approach to generate constant learning.

\section{Results}

Step one of the STBS involves the creation of the value chain (see Fig. 2). These are useful to identify specific components for the value chains and by providing the relevant technologies to be assessed. Consequently, the first step in the STBS process is to investigate the project life cycle so that a generic project value chain can be formulated. In this case, the value chain would initially take the form of a generic bioenergy value chain, which can then be evaluated and expanded to add specific information pertaining directly to the specific case under evaluation. These value chains are an efficient way to generate system- and complexityunderstanding and to communicate this knowledge easily to non-technical individuals.

Within a 'Call for Expression of Interest (EOI)' document, the WfE programme clearly indicated their understanding of the relevant components that form the project value chain. This provided the WfE group with insights relating to the relevant technologies and the four interrelated factors that influence each other:

- Technological Process indicates the conversion processes and the intrinsic technology used. These two factors, process and technology, are inseparable and are thus assessed as a functional unit. The linkage between the technological process and products created is also undeniable as the one determines the other, which must thus also remain within consideration. These factors are easily generated by expert opinion, as they are the available processes required to meet the desired outputs and the project goals. For the Primary Energy Conversion component, four main technological processes were identified due to the overwhelming relevance of these technologies within literature as well as within the market place especially within the EOI. The Technologies proposed were combustion, slow pyrolysis, fast pyrolysis and gasification.

- Technical Specifications are technical aspects that pertain to the technology for only this specific point in the product life cycle or specific value chain component. From an operational point of view, these factors are invaluable to more technical stakeholders as they pertain directly to constraints and challenges, which will be faced. These factors include: complexity of operations, feedstock requirement, residence time and capital cost. Technical Specification focuses on the operational aspects of the Technological Process and may be general or specific.

- Product Requirements creates a linkage between the technological process, its products and their specifications, as required by stakeholders or subsequent processes. This is done to improve the assessment of the technology, as one cannot generate conclusions from the technological process if one does not take aspects and requirements of its products into account. These include the meeting of the stakeholder requirements as well as indicating the various process/product strategies and their affects on sustainability thus the close link 
between the technology, the process, and the product is required to assess performance in relation to the Sustainability Criteria. In this specific case the Product Requirements are difficult to quantify as most of these products are merely subsidiaries and do not directly meet the needs of stakeholders. It is, however, imperative that the stakeholders' needs are considered at this stage so that the correct process/product strategies may be implemented at this early stage to ensure customer satisfaction and ultimately ensure a true reflection of sustainability. The process/product strategy becomes especially important when multiple products and undesirable wastes are produced thus highlighting product benefit trade-offs, as the product number and specifications can be manipulated by changing the process and technical specification. In this case study example it was not deemed necessary to investigate all the various process/product strategies nor all of the products, which could be generated by each general Technological Process. The EOI documents were used as a guide and only products specified within these were assessed, so as to limit the assessment scope as indicated by the stakeholders.

- Sustainability Criteria are key areas that need to be considered for sustainability. Stakeholder engagement and expert opinion is utilised to develop areas for the assessment of the technology in terms of its sustainability. This is the key factor to the STBS and the technology assessment body of knowledge in addressing sustainability. By considering the Sustainability Criteria with the Product Requirements (representing the Product/Process/ Technology complex) matrix in Fig. 3, the clear influence of technology assessment, such as the Multi Criteria Decision Analysis (MCDA) methodologies can be seen. This has been implemented in a simplistic fashion along with the understanding that the Sustainability Criteria and the outcomes generated are likely to form part of an MCDA study to be done after the initial STBS study indicating a strategic direction. The synergies between the STBS and the MCDA are apparent as the STBS facilitates the initial stages of the MCDA cutting down on the time and engagement required by the MCDA but as a rapid assessment tool lacks its quantitative rigour. The STBS focuses qualitative data providing a strategic standpoint through the ranking of factors. Further investigations using strong quantitative data can vindicate the STBS strategic direction and provide further insights. STBS proves valuable in reducing time and costs of a blind MCDA by providing rapid direction and limiting the possibilities assessed by the MCDA, thus limiting the expense of such a time-consuming study. Life Cycle Analysis (LCA) was also investigated and regarded as an excellent tool to further guide decision makers once the STBS had indicated general strategic directions. The LCA decision trees are invaluable to assess process/product strategies that were initially identified by the STBS, quantified by the MCDA and then synthesised by the LCA.

These factors are compared in three assessment matrices to provide insights to the viability and sustainability of the technologies; Fig. 3 provides an example:

- The Technological Process vs. Technical Specification Matrix - evaluates the Technological Process using Technical Specifications to indicate the viability of the various projects and technologies.

- The Technical Process vs. Product Requirement Matrix - evaluates the product aspect pertaining to the ability of the process to provide products that can meet the demands of the market.

- The Technical Process and Product Requirement vs. Sustainability Criteria Matrixevaluates the products that are integral to the Technological Process and the Sustainability Criteria pertaining to the sustainability of the Product/Process. 


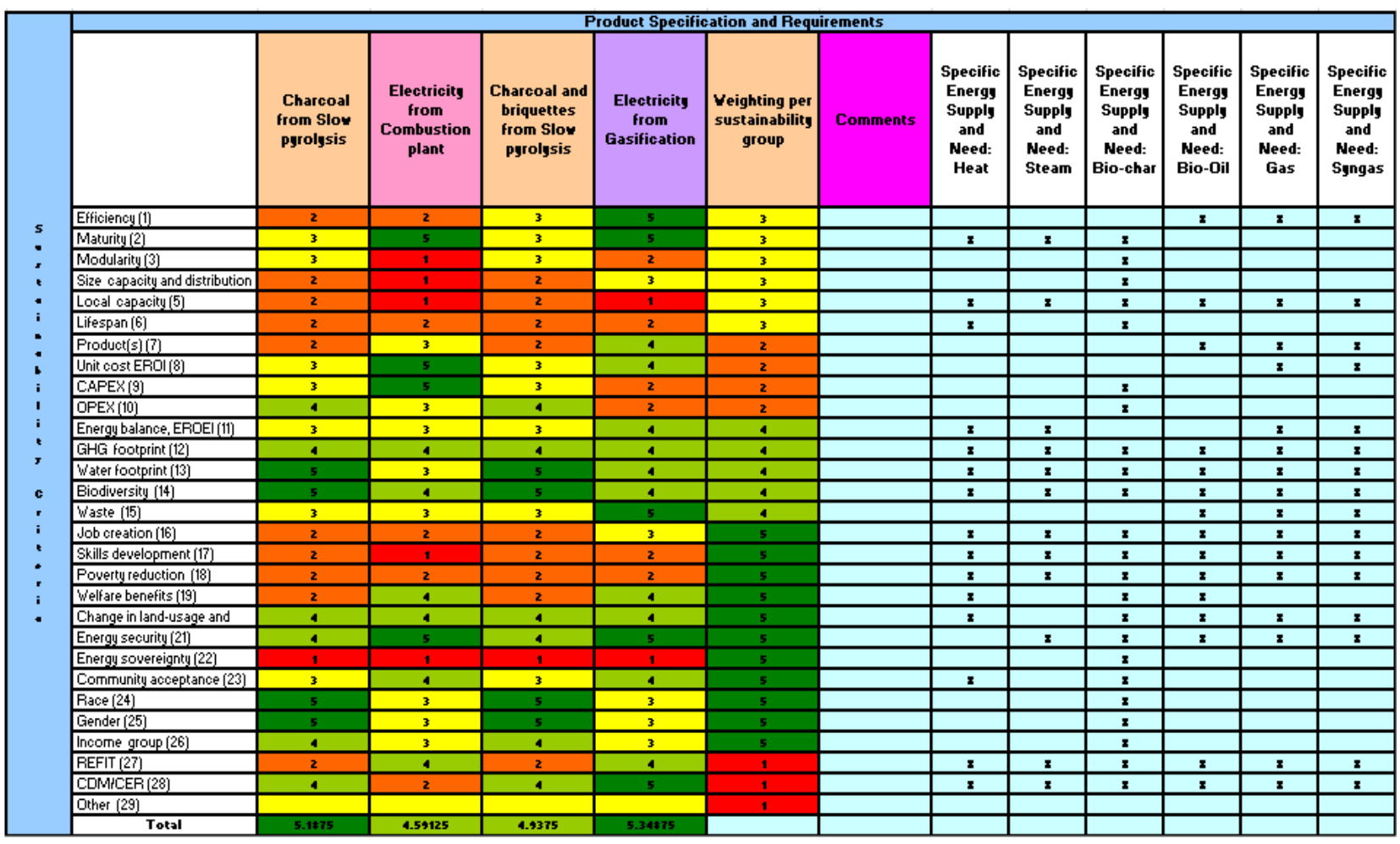

Fig. 3. The Technical Process and Product Requirement vs. Sustainability Criteria Matrix [6].

\section{Conclusions and recommendations}

The recommendations of the WfE stakeholders and experts were diverse, including simple suggestions on framework structure to improve legibility and complex discussions surrounding the communication of STBS factors, driving forces, and the underlying logic of the method. The outcomes included:

- Unambiguous understanding of the conceptual framework and underlying logic, even if the process would still require a facilitation aspect in order to retain integrity.

- A clear buy-in of all the assessment factors in general was communicated and special attention was given to the Sustainability Criteria factor, the formulation of which was deemed to be of critical importance.

- The effectiveness at which the data surrounding the factors where communicated was commended especially the awareness of the Technical Specification factors.

- The strategic intent and direction was intrinsically communicated by the framework.

- The concern surrounding the trade-off between the rapid assessment and the rigour of the assessment was highlighted and it was concluded that the rigour was dependent on the quality of the data used and rate at which the assessment was required. Both factors can be adjusted within the STBS tool to meet the stakeholder requirements.

Thus, the framework itself provides an accurate communication tool aimed at non-technical stakeholders and political decision-makers at various stages in the project life cycle. It provides them with a simple-to-understand strategic direction, a better understanding of the complex system under review using the implementation process insights, which systems thinking provide. This ensures a much improved stakeholder buy-in as well as general "trust brokering”. The framework acts as a high-level cognitive decision tool making use of stakeholders' priorities, and together with the implementation process it is designed to 
compliment and integrate with other tools such as the MCDA and LCA, from which it draws heavily and where the STBS act as a precursor.

The STBS also utilises information generated by other preceding stakeholder engagement tools, thus acting as a truly integrative tool creating a link between other tools and methodologies, which is invaluable to both stakeholders and practitioners alike. In general, expert opinions had been positive in regards to the STBS addressing sustainability, its rapid flexibility and its ease of communication.

As a way forward, the STBS needs further refinement and active development by further case study analyses. The case study requirement is based on specifically utilising the STBS from an early project stage and providing focus for the STBS as the main strategic assessment tool. This would, however, be done in relation to and in close conjunction with other integrative tools developed so as to add value to the STBS and other tools utilised.

\section{References}

[1] H. Bossel, Indicators for sustainable development: Theory, method, applications, A Report to the Balaton Group, IISD, Canada, 1999.

[2] M.W. Pretorius, G. de Wet, A model for the assessment of new technology for the manufacturing enterprise, Technovation 20(1), 2000, pp. 3-10.

[3] G. de Wet, Corporate strategy and technology management: Creating the interface, Graduate School of Technology Management, University of Pretoria, 1992.

[4] H.K. Grover, H.K., M.W. Pretorius, The technology assessment of demand side bidding in the South African context, South African Journal of Industrial Engineering 19(2), 2008, pp. 93-108.

[5] R.K. Singh, H.R. Murty, S.K. Gupta, A.K. Dikshit, An overview of sustainability assessment methodologies, Ecological Indicators 9, 2008, pp. 189-212.

[6] W.D. Peach, The development of the Sustainable Technology Balance Sheet: A generic technology assessment tool to assess the sustainability of renewable energy technologies, Masters dissertation, Graduate School of Technology Management, University of Pretoria, 2010. 